Original Article

\title{
Prospective Study of Harmonic Scalpel in about 800 cases covering both open and laparoscopic methods.
}

\author{
A.M. Shahidulla ${ }^{1}$, Md. Aminul Islam ${ }^{2}$, Zakaria Pavreen ${ }^{3}$ \\ ${ }^{1}$ Professor and Head, Department of Surgery, Dhaka Community Medical College, ${ }^{2}$ Associate Professor, Department of \\ Anaesthesiology, Dhaka National Medical College, ${ }^{3}$ Consultant, Department of Gynae \& Obst., Diabetic Center, Narayangonj.
}

\begin{abstract}
Harmonic (ultracision) Scalpel is a part \& parcel of modern Day surgery, Endo-laparoscopic \& minimally access surgery. We use this instrument in different minimally access surgeries for the last 5 years. Different open \& laparoscopic operations of about 800 cases were performed within this period. Results of our operations with Harmonic Scalpel were very much inspiring, safe and surgeon-friendly with a rapid discharge of the patient from the hospital. The main disadvantages are that the instrument itself is costly $\&$ so is its maintenance. It needs a long term practice to be handy. Among open surgeries breast, thyroid, hemorrhoids, tonsils etc. are to be mentioned. In laparoscopic surgery, Harmonic scalpel reduced total operation time in our series. Harmonic scalpel is a laparoscopic surgical instrument that is changing doctors' performance as well as decreasing patient's recovery time. Although, it is not poor-friendly, but can be used for safe, rapid surgery \& patient-friendly operation.
\end{abstract}

Keywords: Harmonic scalpel, minimally access surgery.

\section{Introduction}

Harmonic Scalpel- the instrument which utilizes ultrasonic energy to enable haemostatic cutting \&/or coagulation of soft tissue. The system (Fig. 1) consists of an ultrasonic generator, foot pedal, hand switch and a variety of open \& minimally invasive instruments. Ultracision instruments vibrate longitudinally at 55.5 Kilohertz.

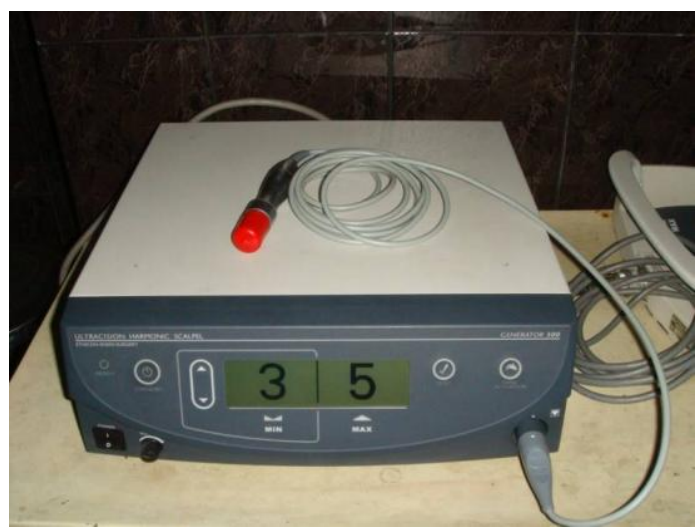

Fig. 1: Photograph of Harmonic Scalpel with its accessories

This ultrasonic vibration at the blade enhances its cutting ability. This same vibration seals same vessels with coagulated blood \& tissue proteins. Haemostasis occurs when tissue couples with the instrument.

These couplings cause collagen molecules within the tissue to vibrate $\&$ become denatured forming a coagulum. Harmonic scalpel is indicated for soft tissue incisions when bleeding control \& minimal thermal injury are desired. It is used both in open \& endoscopic surgical procedures producing less smoke \& heat.

\section{Materials and Methods}

From 2006 to 2011, in about of 5 years duration, 810 cases were undergone surgery using Harmonic Scalpel in the "Day Case Centre", where usually we perform our operations. Out of these operations, maximum were laparoscopic \& a few open cases Non-laparoscopic. Among laparoscopic group Cholecystectomy, Appendectomy, Hysterectomy, Ovarian cystectomy, Ectopic pregnancy, Ureteric calculi, Kidney stone, Herniorrhaphy etc. are to be mentioned (Fig. 2). Among Open group, Lumpectomy \& simple mastectomy of Breast, Thyroid, Tonsil (By ENT surgeons), Perianal operations etc. are to be mentioned. Operations were performed on those patients who were convinced for the higher cost that they have to bear for Harmonic scalpel. Almost all the patients were discharged within one day (day surgery), except a few. So, it was certainly a patient-friendly method.

Patients who were fit for operation and anesthesia were included. No patients were excluded except those who cannot pay for the instrument. Certainly, per-operative $\&$ post-operative recordings were smooth $\&$ refined in comparison to those who were not operated by Harmonic Scalpel. 


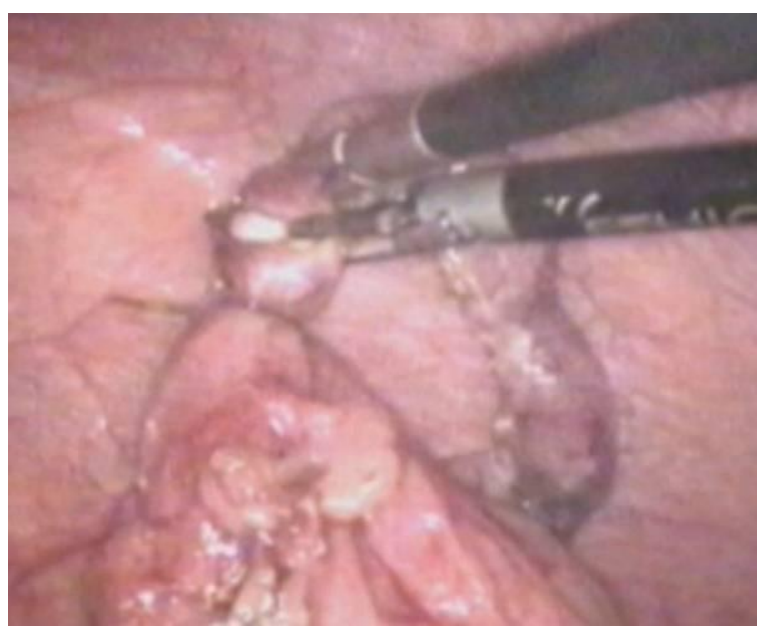

(a) Appendicectomy

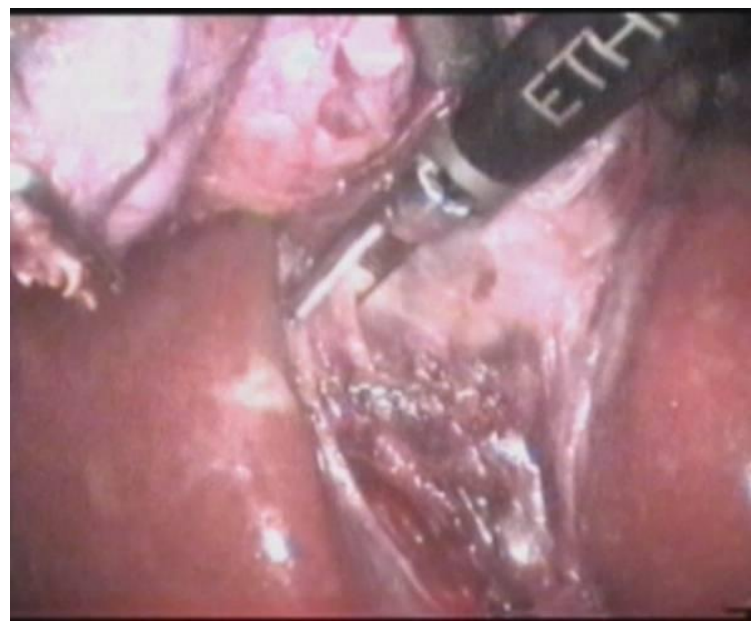

(b) Cholecystectomy

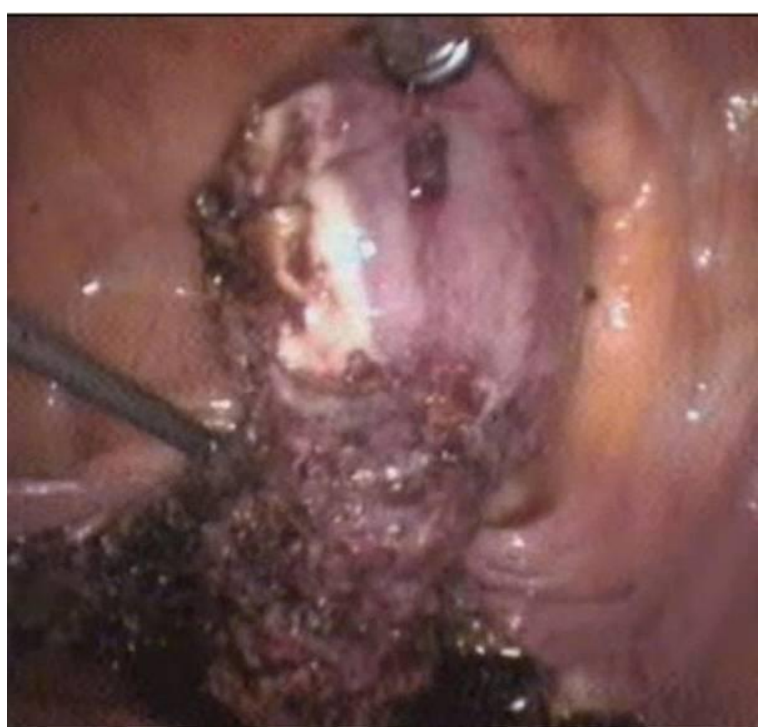

(c) Hysterectomy

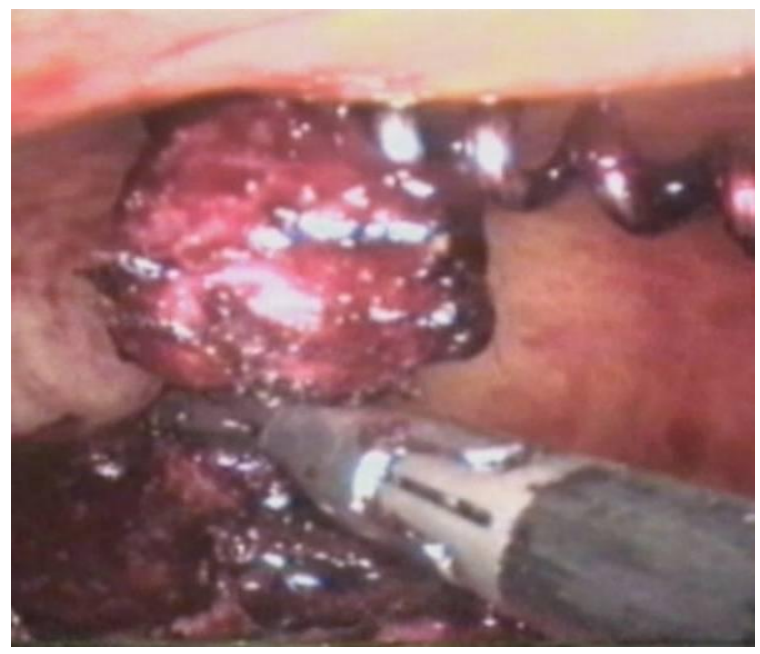

(d) Myomectomy

Fig. 2: Use of Harmonic shear in various operations.

\section{Results}

A total of 810 cases were performed in the "Day Case Centre", where mainly Day \& Laparoscopic surgeries are being done for long 15 years. Use of Harmonic Scalpel has been started for last five years. Both Laparoscopic \& open operations were done by Harmonic Scalpel. Almost equal numbers of male \& female cases were operated. In Laparoscopic methodology, female patients were predominant in Laparoscopic Cholecystectomy \& male patients were predominant in laparoscopic Appendicectomy. In open surgery, breast operation was only done on female patients. Most of the patients recovered on day surgery basis.

No major complication happened except a few minor incidences less important to be mentioned. Male female ratio of total number of patients is shown in Fig. 3. Relative number of patients of two major groups of surgeries undergone in this study by Harmonic Scalpel is shown in Fig: 4. It is to be mentioned here that Anaesthesia did not find any disharmony with these procedures. Both general and regional Anaesthesia could be used. 


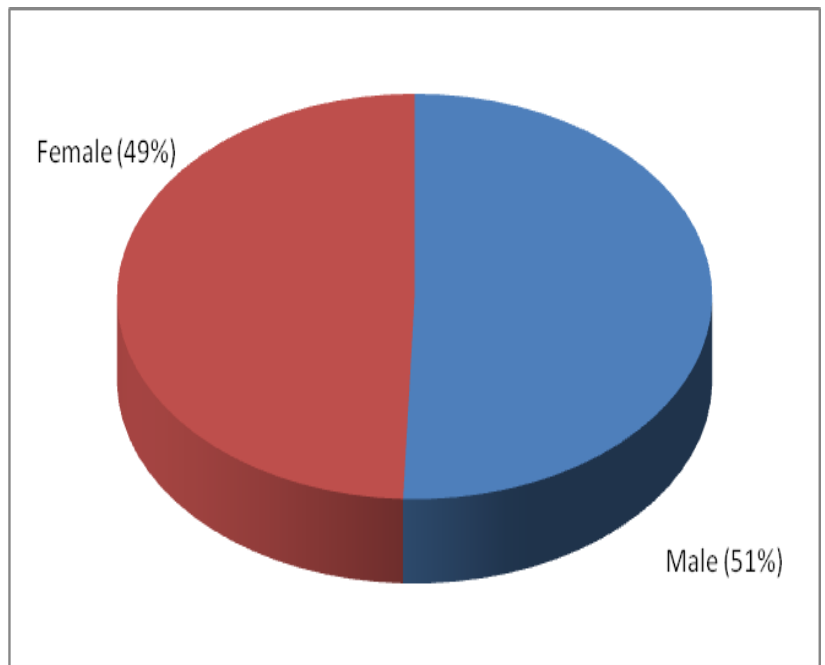

Fig. 3: Sex Distribution of cases

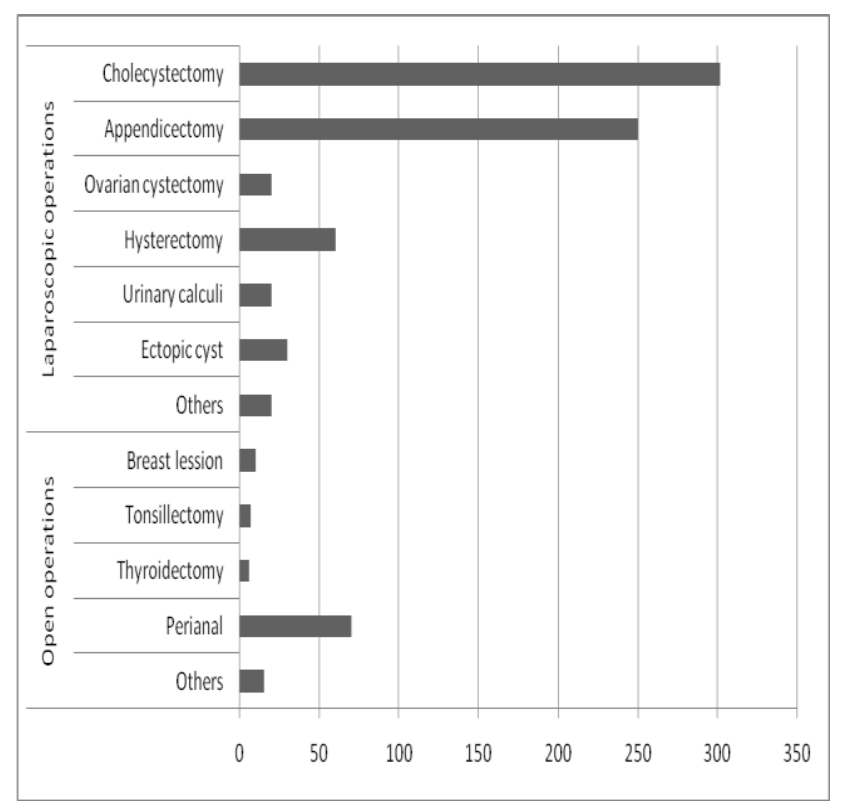

Fig. 4: Percentage of cases harmonic scalpel used

In this study, the advantages and disadvantages of harmonic scalpel are observed and listed below:

\section{Advantages:}

1. Coagulation of arteries of medium size.

2. Coagulation, cutting \& dissection of soft tissue with bleeding control at the same time.

3. Less time of operation.

4. Used both in laparoscopic and open surgery.

5. Blood loss minimum; so, blood is rarely required during operation.

6. Operative field remains clean.
7. Recovery is rapid, good for day surgery and minimally access surgery.

8. Very less heat production, heat necrosis is rare.

9. Bowel can be cut without damage.

10. Good for laparoscopic, pelvic, abdominal, thoracic, perianal, breast, thyroid, tonsil surgery.

11. Surgeon friendly safe surgery.

\section{Disadvantages:}

1. Instrument is costly.

2. Maintenance is also costly; so, not poor friendly.

3. Long term practice is needed for expertise.

4. Delicate soft handling is needed.

5. Incision on bone is not possible.

6. Not intended for contraceptive tubal occlusion.

\section{Discussion}

The main use of Harmonic scalpel (HS)in Laparoscopic Cholecystectomy is to cut Cystic Artery \& avulse the Gall Bladder from its bed in a bloodless field. Advancement of HS blade tip now provide for the reliable ultrasound division \& closing of the cystic duct $^{1}$. In Laparoscopic Appendicectomy, all the early complications of this methodology, such as hemorrhage, caecal burn, fistulae, etc. were overcome by Harmonic scalpel, when it replaced other electric coagulation systems. Not only the mean operation time is reduced but also all varieties of appendicitis anatomical \& pathological could be done safely. There were no complications related to the treatment with UAS (Ultrasonically activated scalpel) of either the vascular pedicle or the appendicular stump ${ }^{2}$.In our study, performing laparoscopic removal of urinary stones were easy, safe in a bloodless field with no reflection of light when HS was used. Laparoscopic nephrectomy, in a study, with Harmonic scalpel was found easy, safe to use; it was cost-effective \& easily controlled bleeding with smooth dissection ${ }^{3}$. In this series, only one case needed to be opened, few needed blood transfusion, which at all not seen when Laparoscopic Hysterectomy was done by Harmonic scalpel. It was observed that use of the Harmonic scalpel to be extremely useful in performing laparoscopically assisted vaginal hysterectomy. Our experiences showed that this can be performed without major complications in a cost-effective manner ${ }^{4}$. A total of 354 consecutive women underwent laparoscopic gynaecological procedures with the UAS \& LCS (Laparoscopic Coagulation Shear), where one major 
complication occurred (bleeding). Results showed that UAS was a safe instrument in gynaecological procedures 5 . Various methods as ultrasonic surgical aspirator (USA) or water jet aspirator \& micro-wave tissue coagulator are used for hepatic dissection. USA and Water jet scalpel can divide the Liver by fracture aspiration of the liver parenchyma but they cannot be used for coagulation or cutting whereas HS can be used for both; thereby reducing the time needed to divide the Liver ${ }^{6}$. Usual time of Tonsillectomy in our study was less than 8 minutes by Harmonic scalpel technique, which was only 5 minutes 52 seconds, in another study having well controlled bleeding \& discharging patient within 12 hours ${ }^{7}$. UAS saves operation time, reduction of pain with less traction during open thyroidectomy ${ }^{8}$. Harmonic scalpel Haemorrhoidectomy \& related perianal surgeries in our study compares similarly in respect of operation time, haemostasis, safety \& smooth recovery with another study ${ }^{9}$. In breast surgery, in our series except radical mastectomy, all were converted into day surgery $\&$ astonishingly no one needed drainage tube with healing of wound by cosmetic intradermal stitches. Harmonic scalpel in mastectomy significantly reduces blood loss, postoperative pain, total drainage volume and days but does not lower operative time, seroma, total hospital stay and till now cannot be considered cost effective ${ }^{10}$. Harmonic Scalpel does not rely on the flow of current through the patient, so it is of safety when surgeons are working near central vascular catheters or pacemaker wires. It is also safe during important defibrillation devices \& no disturbances with monitoring systems, so improves patient's safety. All the above measures are needed for long term safe anaesthesia ${ }^{11}$.

\section{Conclusion}

From the above results \& discussions, Harmonic scalpel has been considered as a safe latest surgical instrument that is changing surgeons' performances to refined, quicker \& efficient procedures. Apart from reduction in operation time than the conventional, Harmonic scalpel also allows the patients to take less anesthetic drugs \& its related hazards. Its only disadvantage is that it is not cost-effective for a poor country like us.

\section{References}

1. Nazih Salameh Amarin; "Harmonic Scalpel and Clipless Cholecystectomy", World Journal of Laparoscopic Surgery, May-August 2008; 1(2): 6-8.

2. JC Martín del Olmo, JI Blanco Álvarez, MA Carbajo Caballero, et al. Laparoscopic Appendectomy by Ultrasonically Activated Scalpel in Acute Appendicitis: Preliminary Report; Journal of Laparoendoscopic \& Advanced Surgical Techniques, July 7, 2004: 12(2): 111-113.

3. Yung-Tai Chen, Stephen Shei Dei Yang, Cheng-Hsing Hsieh. Hand Port-Site Metastasis of Renal-Cell Carcinoma Following Hand-Assisted Laparoscopic Radical Nephrectomy: Case Report. Journal of Endourology, November 2003; 17(9): 771-775.

4. ML Winter, SA Mendelsohn. Total Laparoscopic Hysterectomy Using the Harmonic Scalpel. JSLS, Journal of the Society of Laparoendoscopic Surgeons, July-September 1999; 3(3):185-186(2).

5. FW Jansen, TT Kemper, JB Trimbos. Ultrasonic scalpel in laparoscopic gynaecological surgery: an observational study in 354 cases. Journal of the Gynaecological Endoscopy, February 2002; 11(1): 47-51.

6. Hiroyuki Sugo, Youshi Mikami, Fumio Matsumoto et al. Hepatic Resection using the Harmonic Scalpel. Surgery Today, 2000; 30: 959-962.

7. Video: KT Kavanagh. Tonsillectomy harmonic scalpel technique. Kevin T Kavanagh.

8. P Miccoli, P Berti, GL Dionigi. Randomized Controlled Trial of Harmonic Scalpel Use During Thyroidectomy. Archieve of Otolaryngol Head Neck Surgery, 2006; 132: 1069-1073.

9. DN Armstrong, C Frankum, ME Schertzer, et al. Harmonic Scalpel® Hemorrhoidectomy Five Hundred Consecutive Cases. Diseases of the Colon \& Rectum, 45(3):354-359.

10. A Khater. Harmonic scalpel as a single instrument in modified Radical Mastectomy. Is it more cost effective than electrocautery and ligature? Egyptian Journal of Surgery, April, 2010; 29(2): 959-962

11. Paredes J, Borges M, Coulson A. Early impressions of the harmonic scalpel. Anaesthesiology, Oct. 1996; 85(4):947-950. 